\title{
Optimization of Physical Activity as a Countermeasure of Bone Loss: A 5-Year Study of Bikram Yoga Practice in Females
}

\author{
Sophia N. Sangiorgio',2, Arnob K. Mukherjee3, Nicole W. Lau', Apurba Mukherjee, \\ Prithwis Mukhopadhyay3, Edward Ebramzadeh",2 \\ ${ }^{1}$ Orthopaedic Biomechanics and Mechano-Biology Laboratory, Orthopaedic Institute for Children, Los Angeles, \\ USA \\ ${ }^{2}$ Department of Orthopaedic Surgery, University of California, Los Angeles, USA \\ ${ }^{3}$ Bikram's Yoga College of India, Los Angeles, USA \\ ${ }^{4}$ University of Southern California, Los Angeles, USA \\ Email: Sophia.biomechanics@gmail.com
}

Received 22 March 2014; revised 28 April 2014; accepted 6 May 2014

Copyright (C) 2014 by authors and Scientific Research Publishing Inc.

This work is licensed under the Creative Commons Attribution International License (CC BY).

http://creativecommons.org/licenses/by/4.0/

(c) (i) Open Access

\section{Abstract}

Over the last decade, the number of people diagnosed with osteoporosis has increased dramatically due to many factors. While it has been asserted that one variable associated with preserving bone health is participation in high-impact exercises, those same exercises are also well documented to damage the hip and knee joints. Therefore, the goal of the present study was to evaluate the potential benefits of a highly regimented, low impact weight-bearing exercise, Bikram Yoga. Specifically, the bone mineral content was measured using DEXA analysis of the proximal femur and lumbar spine of nine female Bikram Yoga instructors between the ages of 30 and 59, who not only participated in a minimum of 3 classes (4.5 hours) a week, but also actively taught Bikram Yoga classes. These same participants, who remained active as instructors and practitioners, were rescanned five years later. The results of the study, which focused on the percent change in individual bone mineral density over the five-year period were as follows: the premenopausal subjects showed a mean increase in BMD of $6.6 \%$ at the femoral neck, $2.0 \%$ for the total hip, and $1.0 \%$ for the total lumbar spine. In contrast, post-menopausal subjects revealed a mean decrease in bone mineral density (BMD) of $-6.0 \%,-8.1 \%$, and $-5.6 \%$ in the femoral neck, total hip, and total lumbar spine, respectively. Consequently, the results of this study suggest that performing Bikram Yoga may preserve, or even perhaps, increase bone mineral density in pre-menopausal women and hence, may be an effective countermeasure for preventing osteoporosis. 


\section{Keywords}

\section{Osteoporosis, Bone Mineral Density (BMD), Exercise}

\section{Introduction}

The relationship between poor bone quality and the onset of osteoporosis has been widely reported in recent years. The National Osteoporosis Foundation (NOF) has reported that 44 million Americans have osteoporosis and by 2020, nearly 50\% of Americans over the age of 50 are anticipated to have low bone density or osteoporosis [1]. It has been well established that a decrease in bone mineral density correlates to an increased risk of bone fractures, commonly known as fragility fractures [2] [3].

These fragility fractures have also been associated with increased health care costs, and decreased quality of life [4]. Thus, finding viable solutions to increase prevention of osteoporotic fractures highlights the importance of identifying effective methods to preserve bone density and avoid osteoporosis. While factors such as aging, gender, ethnicity, and genetics greatly determine a person's bone mineral density, there are other factors that can affect BMD that are within a patient's control. For example, multiple risk factors and lifestyle choices have been identified to influence bone mineral density.

Over the last decade, researchers have noted that one of the most effective ways to take preventative measures against fragility fractures is to engage in physical activity, as it helps to increase bone mineral density (BMD) by efficiently building bone mass [5], much in the same way that physical activity helps to increase muscle mass. Incorporating exercise into daily routines has been observed to correlate to increases in BMD. More specifically, researchers have discovered that weight-bearing exercises have the potential to bone mineral density [6]. Weight-bearing exercises such as running and jogging, for example, have demonstrated more substantial increases in bone density than other types of physical activity. Some of these potential benefits to maintaining BMD have been quantified, reporting decreases in the rate of bone mineral density deterioration [7].

Unfortunately, many of these high intensity exercises that previous studies found to be most advantageous for increasing bone density are also some of the most difficult types of physical activities for at risk and elderly patients to engage in. High impact activities such as basketball and volleyball are not always viable options for the elderly and aging because many of them already have osteoporosis or are physically incapable of such vigorous movements. Furthermore, while high impact exercises help to increase BMD, they also have other physical downsides. Specifically, those who engage in rigorous weight-bearing exercises also increase their chances of developing joint-related disorders, such as osteoarthritis [8]. Additionally, low-impact forms of exercise fail to ameliorate the problem of decreased bone density. Specifically, exercises such as swimming and cycling offer the benefits of increased circulation and improved overall health, however, participation in these activities has failed thus far to demonstrate the ability to increase BMD through muscle action alone, and has actually suggested decreases in bone mineral density [9]-[13]. Therefore, there is a clear need for a type of exercise capable of both protecting the joints and preserving bone density to address this osteoporotic dilemma.

The goal of the present study was to evaluate the effects of a highly regimented form of a low-impact weight-bearing physical activity, Bikram Yoga, in a well-controlled population. Fourteen Bikram Yoga instructors who actively practiced a minimum of three times a week (90 minutes per session) and instructed classes regularly were recruited to have bone density scans (DEXA) five years ago. These results were reported previously [14]. Of the initial fourteen subjects, nine, all of whom continued practicing Bikram Yoga a minimum of three times per week and continued to instruct classes regularly, were available to return to the Bone Density Lab, for 5-year follow-up DEXA scan. The hypothesis of this study was that Bikram Yoga would serve as an effective measure to preserve, or ideally, increase bone mineral density without causing the joint-related side effects reported by athletes who participated in high-impact activities.

\section{Study Methods}

\subsection{Inclusion Criteria}

In 2005, subjects were recruited from Bikram's Yoga College of India in Los Angeles. Inclusion criteria in- 
cluded females between the ages of 30 and 59, who were certified Bikram Yoga instructors that had been practicing for a minimum of three years. All participants were in good physical health, capable of several hours of instruction a day, and performing and demonstrating proper technique for exercises, which require above average strength and stamina. Fourteen subjects were included and of those, nine subjects fit the inclusion criteria and were available to return to the same Bone Density Clinic for five-year follow-up measurements [14] (Table 1).

\subsection{Measurements}

All nine patients underwent bone density assessments at the Los Angeles Orthopaedic Hospital Bone Density Clinic. Dual Energy X-Ray Absorptiometry (DEXA) was used to quantify BMD. Measurements were made using a Hologic 2000 bone densitometer (Hologic, Inc., Waltham, MA), an enhanced form of X-ray technology. The same operator took all measurements, with patients positioned with both legs internally rotated 15 degrees for femoral measurements.

Three separate bone density scans were completed at the initial and five year follow-up visit: 1) Proximal Femur, 2) Lumbar Spine, and 3) Total Body. In addition to these global measurements, the following regions of interest were selected for analysis using the Orthopaedic Software (Hologic, Inc., Waltham, MA): femoral neck, Ward's region, intertrochanteric region, greater trochanter, total hip, L1, L2, L3, L4, and total lumbar spine (L1 L4 combined, with L5 excluded).

In addition to bone mineral density, T-scores and Z-scores were reported. The T-score used was a comparison of a patient's BMD to that of a healthy thirty-year-old of the same sex, assumed to have peak bone mass. Tscores were used to determine fracture risk, with a T-score of 1.0 indicating that the subject's bone density is one standard deviation above the average 30 year old, with -1.0 indicating one standard deviation below the average. Typically, a T-score of -1.0 to -2.5 was often associated with a diagnosis of osteopenia, or low bone density. A score of -2.5 or lower was typically considered to indicate an increased risk of fragility fractures, or if one had already occurred, osteoporosis. Additionally, Z-scores were calculated similarly, but were normalized to a population of the same age, gender, and ethnicity for each unique subject.

\subsection{Data Analysis}

For each subject, the change in bone mineral density in each of the following regions of interest was calculated: femoral neck, Ward's area, intertrochanteric region, greater trochanter, total hip, and total lumbar spine. This change was expressed in two ways: 1 ) by subtracting the more recent measurements from the corresponding initial measurement and 2) by dividing this difference by the initial measurement and then subtracting 1 , to reflect the percentage increase or decrease in bone density as a positive or negative number. More attention was paid to raw $\mathrm{BMD}$ measurements rather than $\mathrm{Z}$ and $\mathrm{T}$ scores due to potential inconsistencies in the $\mathrm{Z}$-score data.

The ages of the participants at the last follow up varied from 37 to 64, with half of the women falling in the postmenopausal category. As it has been previously established that the rate of bone density loss accelerates following the onset of menopause, the women were divided into two age groups for comparison purposes: 1) pre-menopausal and 2) post-menopausal. Individual two-tailed Student's t-tests were conducted, assuming equal variances in both groups, to compare mean percent change in each region of interest for pre-menopausal women to that of postmenopausal women.

\section{Results}

The bone mineral density from the DEXA scans from each of the following regions: femoral neck, Ward's region, intertrochanteric region, greater trochanter, total hip and total lumbar spine (L1 - L4 combined). These regions were selected because these areas represented the highest risk regions for fractures in osteoporotic patients. Specifically, femoral neck region has the highest risk of fracture due to simple falls, and the lumbar spine region has the highest risk for compression fractures, which can occur without trauma or warning. Representative scans of the femur (Figure 1) and the lumbar spine (Figure 2) from a healthy subject included in this study are provided for reference. The percent change in BMD over the five-year study period was calculated for each region and each subject (Table 2). As shown, negative percentages indicate a drop in bone mineral density, whereas positive percentages indicate an increase.

After the women were separated into two categories, pre-menopausal and postmenopausal, several trends became apparent. Mean change BMD in the femoral neck for pre-menopausal women showed an increase of $6.6 \pm$ 
Table 1. Demographics of the study group.

\begin{tabular}{ccccc}
\hline & \multicolumn{4}{c}{ Demographics } \\
\hline Subject & Age at 5 year DEXA scan & Height (inches) & Weight (lbs) & Ethnicity \\
\hline Subject \#1 & 37 & 65 & 134 & Caucasian \\
Subject \#2 & 45 & 65 & 135 & Asian \\
Subject \#3 & 52 & 66 & 127 & Caucasian \\
Subject \#4 & 52 & 64 & 160 & Caucasian \\
Subject \#5 & 54 & 64 & 107 & Asian \\
Subject \#6 & 55 & 61 & 102 & Caucasian \\
Subject \#7 & 52 & 66.5 & 130 & Caucasian \\
Subject \#8 & 64 & 63 & 125 & Caucasian \\
Subject \#9 & 46 & 64.5 & 110 & Caucasian \\
\hline
\end{tabular}

Table 2. Percent change in bone mineral density for each subject in each region of interest over the five year study period.

\begin{tabular}{|c|c|c|c|c|c|c|}
\hline \multicolumn{7}{|c|}{ Percent Change in BMD over 5 Years of Intense Bikram Yoga Practice } \\
\hline & Neck & Ward's & Inter & Trochanter & Total Hip & Total Lumbar \\
\hline Subject \#1 & $0.1 \%$ & $-5.1 \%$ & $-1.6 \%$ & $-5.0 \%$ & $-2.7 \%$ & $-5.4 \%$ \\
\hline Subject \#2 & $4.6 \%$ & $1.8 \%$ & $1.4 \%$ & $0.6 \%$ & $2.2 \%$ & $2.3 \%$ \\
\hline Subject \#3 & $-5.2 \%$ & $-5.5 \%$ & $-9.3 \%$ & $-15.0 \%$ & $-11.3 \%$ & $-10.5 \%$ \\
\hline Subject \#4 & $8.9 \%$ & $7.7 \%$ & $6.5 \%$ & $5.6 \%$ & $6.6 \%$ & $5.7 \%$ \\
\hline Subject \#5 & $3.2 \%$ & $0.6 \%$ & $2.1 \%$ & $3.0 \%$ & $2.4 \%$ & $4.7 \%$ \\
\hline Subject \#6 & $-14.7 \%$ & $-20.7 \%$ & $-11.3 \%$ & $-14.5 \%$ & $-12.5 \%$ & $-12.9 \%$ \\
\hline Subject \#7 & $-9.3 \%$ & $-13.8 \%$ & $-10.1 \%$ & $-14.9 \%$ & $-10.9 \%$ & $-13.0 \%$ \\
\hline Subject \#8 & $-4.0 \%$ & $-12.5 \%$ & $-10.5 \%$ & $-7.4 \%$ & $-8.4 \%$ & $3.9 \%$ \\
\hline Subject \#9 & $13.0 \%$ & $16.6 \%$ & $0.3 \%$ & $5.4 \%$ & $1.9 \%$ & $1.3 \%$ \\
\hline
\end{tabular}
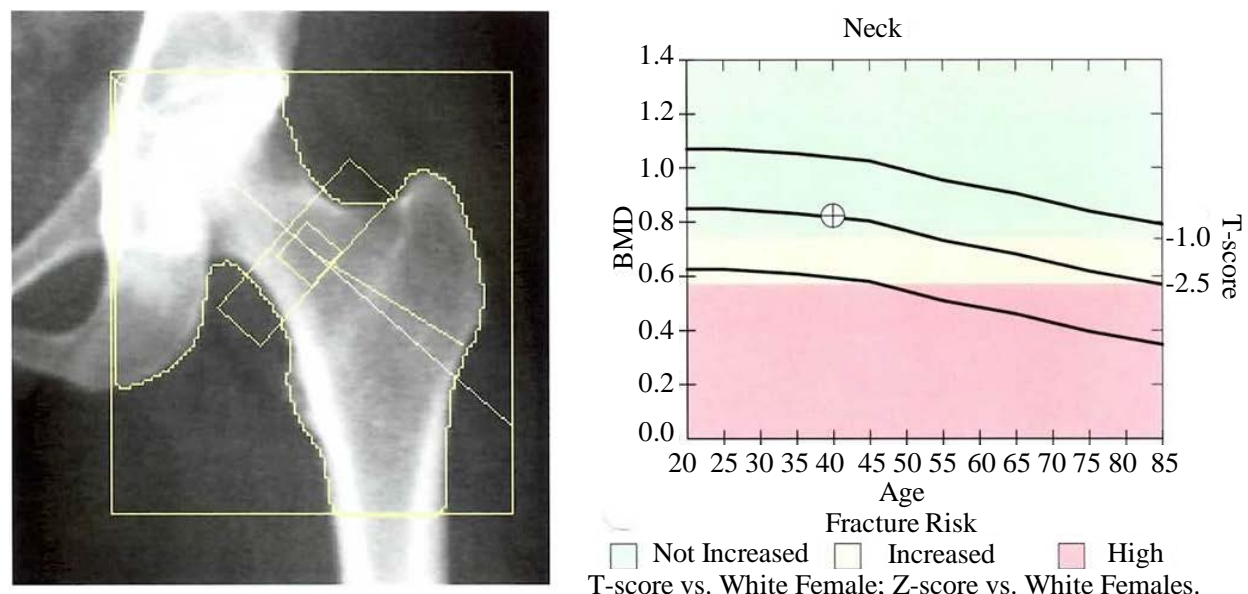

DXA Results Summary:

T-score vs. White Female; Z-score vs. White Females.

\begin{tabular}{|c|c|c|c|c|c|c|c|}
\hline Re & $\begin{array}{l}\text { Area } \\
\left(\mathrm{cm}^{2}\right)\end{array}$ & $\begin{array}{c}\text { BMC } \\
\text { (g) }\end{array}$ & $\begin{array}{l}\text { BMD } \\
\left(\mathrm{g} / \mathrm{cm}^{2}\right)\end{array}$ & $\begin{array}{c}\text { T- } \\
\text { score }\end{array}$ & $\begin{array}{l}\text { PR } \\
(\%)\end{array}$ & $\begin{array}{c}\text { Z- } \\
\text { score }\end{array}$ & $\mathbf{A M}$ \\
\hline & & 3.61 & 0.822 & -0 . & 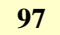 & 0. & \\
\hline & & 6.24 & & 0. & & -0 . & \\
\hline & 1 & 8.8 & & 0.0 & 10 & 0.1 & 0 \\
\hline & 3 & 28.70 & 0.9 & -0 & 9 & 0.0 & \\
\hline ard's & 1.30 & 0.96 & 0.742 & 0.1 & 10 & 0.7 & 1 \\
\hline
\end{tabular}

Total BMD CV 1.0\%, ACF $=1.026, \mathrm{BCF}=1.014, \mathrm{TH}=5.675$

WHO Classification: Normal

Fracture Risk: Not Increased

Figure 1. DEXA scans of hip. 

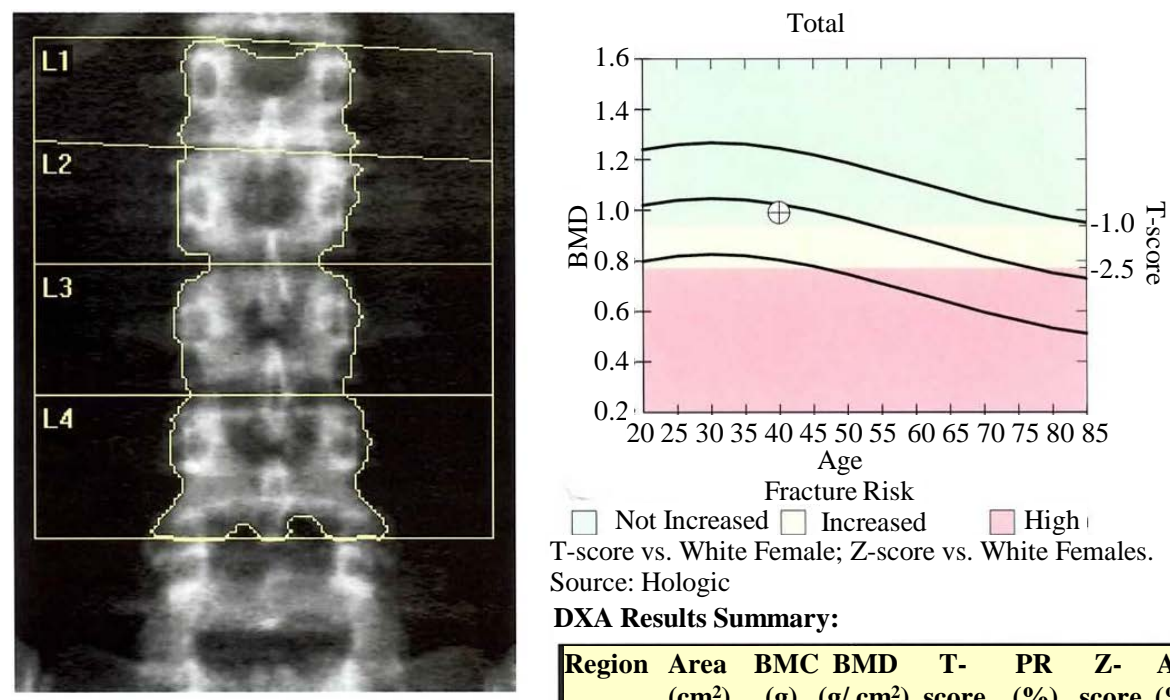

T-score vs. White Female; Z-score vs. White Females.

Source: Hologic

DXA Results Summary:

\begin{tabular}{|c|c|c|c|c|c|c|c|}
\hline Region & $\begin{array}{l}\text { Area } \\
\left(\mathrm{cm}^{2}\right)\end{array}$ & $\begin{array}{l}\text { BMC } \\
\text { (g) }(\end{array}$ & $\begin{array}{l}\text { BMD } \\
\left(\mathrm{g} / \mathrm{cm}^{2}\right)\end{array}$ & $\begin{array}{c}\text { T- } \\
\text { score }\end{array}$ & $\begin{array}{l}\text { PR } \\
\text { (\%) }\end{array}$ & $\begin{array}{c}\text { Z- } \\
\text { score }\end{array}$ & $\begin{array}{l}\text { AM } \\
\text { (\%) }\end{array}$ \\
\hline L1 & 11.02 & 11.20 & 1.016 & 0.8 & 110 & 1.0 & 112 \\
\hline L2 & 11.98 & 11.76 & 0.982 & -0.4 & 95 & -0.2 & 98 \\
\hline L3 & 14.14 & 13.44 & 0.950 & -1.2 & 88 & -1.0 & 90 \\
\hline L4 & 17.68 & 17.91 & 1.013 & -0.9 & 91 & -0.7 & 93 \\
\hline Total & 54.83 & 54.30 & 0.990 & -0.5 & 95 & -0.3 & 97 \\
\hline
\end{tabular}

Total BMD CV 1.0\%, ACF = 1.026, BCF = 1.014, TH = 6.618

WHO Classification: Normal

Fracture Risk: Not Increased

Figure 2. DEXA scans in the spine.

5.5 , whereas for postmenopausal women, mean change of BMD in the same region was $-6.0 \pm 6.6$, representing a loss of BMD (Table 3). Trends were similar in the lumbar spine, though not as striking. For example, the mean increase in lumbar BMD in the premenopausal women was $1.0 \pm 4.7$, whereas in postmenopausal women, the mean change was $-5.6 \pm 9.1$, representing a loss of BMD (Table 3).

\section{Discussion}

The primary goal of this study was to quantify the change in bone mineral density over a five-year period in women practicing a highly regimented form of low impact, weight-bearing exercise, Bikram Yoga. It is significant to note that subjects were of a select group-all were trained instructors of Bikram Yoga and had been performing yoga a minimum of three times per week, several for more than 20 years. The results showed an increase in average bone mineral density at all sites scanned with DEXA among premenopausal subjects, and an overall decrease in bone mineral density for postmenopausal subjects. Overall, the data from this study suggests that participation in Bikram Yoga may provide an increase in bone mineral density in premenopausal women, but an expected rate of bone loss in postmenopausal women would still remain. While it is uncertain whether or not Bikram Yoga can effectively combat osteoporosis on its own, many previous studies have shown that exercise in combination with the proper diet and supplements has been shown to increase BMD, specifically in those with preexisting osteopenia or osteoporosis [15]-[18]. Due to its numerous health benefits and accessibility to an aging and postmenopausal populations, Bikram Yoga is an effective form of exercise that may benefit the overall health of its participants.

Other studies investigating the effects of low impact exercise on bone density have reported similar findings. Specifically, one study focusing on yoga's effect on bone mineral density also compared osteopenic —at risk of osteoporosis-and osteoporotic patients that participated in yoga for two years to a less active control group [19]. At the end of the two years, subjects who had done 10 minutes of yoga daily showed an increase in both spine (0.6) and hip (0.87) BMD using the T-scale to compare changes. Conversely, control values reported a loss of 0.12 and 0.07 in the spine and hip, respectively. In another study, researchers investigated the effects of a similar weight bearing, low-impact exercises, Tai Chi and Resistance Training [20]. Although the male patients' bone density did not benefit as greatly from the Tai Chi, the female patients' hip T-scores from the Tai Chi and Re- 
Table 3. Mean and standard deviation of the percent change over five years for the pre-menopause and post-menopause groups. The P-value is the significance of the difference in percent change between the two groups.

\begin{tabular}{ccccccccccccc}
\hline & \multicolumn{2}{c}{ Neck } & \multicolumn{2}{c}{ Ward's } & \multicolumn{2}{c}{ Inter } & \multicolumn{2}{c}{ Trochanter } & \multicolumn{2}{c}{ Total Hip } & \multicolumn{2}{c}{ Total Lumbar } \\
\cline { 2 - 13 } & Mean & Std Dev & Mean & Std Dev & Mean & Std Dev & Mean & Std Dev & Mean & Std Dev & Mean & Std Dev \\
\hline Pre-Menopause & $6.6 \%$ & $5.5 \%$ & $5.2 \%$ & $9.2 \%$ & $1.6 \%$ & $3.5 \%$ & $1.6 \%$ & $5.0 \%$ & $2.0 \%$ & $3.8 \%$ & $1.0 \%$ & $4.7 \%$ \\
Post-Menopause & $-6.0 \%$ & $6.6 \%$ & $-10.4 \%$ & $8.2 \%$ & $-7.8 \%$ & $5.6 \%$ & $-9.8 \%$ & $7.8 \%$ & $-8.1 \%$ & $6.1 \%$ & $-5.6 \%$ & $9.1 \%$ \\
P-Value & \multicolumn{2}{c}{0.02} & \multicolumn{2}{c}{0.03} & \multicolumn{2}{c}{0.02} & & 0.04 & & 0.02 & & 0.24 \\
\hline
\end{tabular}

sistance Training groups showed an increase of 0.07 and 0.09 , respectively [20]. The results of this study suggested that low weight-bearing exercise is beneficial for increasing female BMD. As seen in two previous studies, the findings suggest that low-impact exercises like yoga and Tai Chi can benefit those with preexisting osteoporotic conditions.

More specifically, Bikram Yoga is a unique form of yoga practice that has a highly regimented exercise routine that may help to combat female osteoporosis due to its effects on increasing BMD in premenopausal women and lessening BMD decrease in postmenopausal women. The postures and the duration of each held position are all highly controlled, and monitored by instructors who are required to undergo routine re-certification. Additionally, Bikram Yoga is practiced in a humidity controlled studio at an ambient temperature of $105^{\circ}$, which also affects circulation, respiration, and perspiration. With all these strict regiments, those subjects who have been practicing Bikram Yoga for over twenty years have been accustomed to this stress and intensity of exercise. Furthermore, the familiarity of our participants to typical Bikram Yoga parameters-intense poses, routines, and ambient temperature-may been the reason there was only a slight observations increase in BMD results. Based on this notion, this may explain why premenopausal group may have experienced a greater increase in BMD not only due to their age, but perhaps also due to required stamina and familiarity with the unique demands of Bikram Yoga.

In regards to the decreased BMD of the postmenopausal group, it is difficult to determine how this loss compares to the expected loss in BMD experienced by post-menopausal women. It has been widely reported that peak bone mass in females is achieved during the teenage years and later plateaus in early twenties. After this plateau, age-related bone loss occurs at a rate of approximately $0.5 \%-1.0 \%$ per year, which tends to accelerate upon the onset of menopause, increasing to a rate of $1.0 \%-2.0 \%$ each year [21]. Researchers also noted that T-scores varied at various locations in large-scale populations, plotting the rate of decline with increasing age as a function of race or ethnicity [21]. For instance, the peak bone mineral density of the femoral neck in Caucasian females decreased roughly one full T-score between the ages of 40 and 60 [21]. Other researchers also confirmed that that bone mineral density remains relatively stable between 20 and 40 -years-of-age, and then decreased markedly for women during peri-menopause and menopause; by the time a woman has reached her eighties, she has a fifty percent chance of having osteoporosis [22] [23]. Taking such statistics into consideration, the decline in BMD found in the post-menopausal subjects fell within the expected range for women in their age category.

In order to combat the deleterious effects of menopause and BMD decreases, the optimal form of exercise must not only help build up BMD but also be accessible. For instance, numerous studies have been published asserting that high-impact exercises increase bone mineral density. One such study that exemplifies this idea chose to focus on female college athletes in various sports [10]. The athletes were then divided into three groups based upon the amount of impact based on ground reaction forces associated with their sport. Basketball and volleyball comprised the high-impact group; soccer and middle- to short-distance track competitors comprised the medium-impact group; swimmers comprised the non-impact group; and individuals exercising less than one hour/week comprised the control group. The main findings from this study displayed that athletes involved in the highest impact sports had the highest bone mineral densities at weight-bearing sites. Furthermore, not only did swimmers from the non-impact group show significantly less bone mineral density at the hip than those who participated in high-impact sports, but also their BMD values were no different than those from the sedentary control group. Previously conducted studies also highlight that the bone status in professional cyclists discovered that elderly, professional cyclists had significantly lower whole body BMD than the sedentary control group [9]. 
Thus, for postmenopausal women especially, it becomes increasingly important to choose a method of exercise that is most accessible while still being beneficial to bone health. While high impact exercises offers the most benefits in regards to BMD, there are joint complications that may arise that affect the feasibility of sustained exercise in elderly population [8]. Although low impact exercise is often more accessible, it has been shown to have negative effects on BMD as seen with the previous two studies mentioned due to the minimal weight bearing exercise taking place. Therefore, a weight bearing, low impact form of exercise such as Bikram Yoga may offer an intermediate solution to maintaining overall health through exercise, preserving or improving bone density, and remaining feasible to perform for an elderly population.

While few studies have attempted to quantify the benefits of Bikram Yoga on bone health, others have reported improvements in quality of life and physical adeptness. Specifically, investigators have compared data from an experimental group that practiced yoga and a sedentary control group [24]. The results highlighted how participation in the yoga program directly correlated with improved measurements of quality of life based on improved social functioning, higher vitality and energy, and less fatigue and bodily pain [24]. Furthermore, in other studies examining the therapeutic effects of yoga, researchers further outlined the benefits of practicing yoga, which included enhanced muscular strength and flexibility, improved respiratory and cardiovascular function, and reduced psychological stresses [25]. Previous researchers have found convincing evidence that the amount of blood flow to lower extremities directly affects BMD and fragility fractures [26]. This study offered suggestive evidence that underscored how women with increased blood flow to the lower extremities had a higher bone mass at the hip and in the appendicular skeleton. It was also observed that women with decreased blood flow in the lower extremities had a larger rate of bone loss than those with a higher measured blood flow [26].

While our present study yields insightful information regarding the potential benefits of Bikram Yoga on bone mineral density, there were several limitations to our studies. First off, no control group was included. There were several reasons for this decision, the most important being that the authors felt it would be unethical to ask a control group of women to remain inactive, not participating routinely in any sort of physical activity or sport for five years in order to serve as true controls. Secondly, because of the immense databases of large sample populations used by DEXA machine manufacturers to calculate T- and Z-scores for each patient, we felt that Z-scores would serve as the most reliable controls. It was publicly available and it specifically listed patients of every age, gender, and ethnicity.

Another limitation to the present study was the small experimental pool. While all 14 of the original instructors were available by phone and reported no bone or joint-related fractures or disorders, only nine were able to return to the Bone Density Clinic for follow-up scans on the same machine. As different DEXA manufacturers define regions of interest (such as the femoral neck) using different bony landmarks, and patient populations that are used to generate T- and Z-scores vary not only by machine but by software versions, it is very important to use the same machine, software, and technician, if possible, when comparing changes in bone density over a short period. Therefore, simply asking patients who were unable to return to the same clinic to visit another DEXA site was not considered valid.

In future studies, more patients meeting certain conditions will be recruited for initial scans to increase the probability of having more available patients to return for later scans. Furthermore, more patients with pre-existing osteopenia or osteoporosis conditions will be recruited in order to see if exercise is actually beneficial to those with even lower BMD. Particularly, recruiting patients with varying levels of Bikram Yoga experience should also included, as those who have been performing Bikram Yoga for less time may experience greater benefits than experienced instructors, some of whom have been practicing for over 20 years. Most importantly, having a larger experimental group will allows researchers to draw more definitive results about the relationship between Bikram Yoga and osteoporosis. Future studies will need to include a control group of less active patients who are of the same age, gender, and ethnicity to the experimental pool. Because this present study did not seek to quantify any changes, beneficial or detrimental, to joint health, future studies will likely include such analysis.

\section{Practical Applications}

The findings from the present study suggest that Bikram Yoga may be a viable form of exercise to help increase or preserve bone density in the pre-menopausal population, and help to mitigate bone loss in the post-meno- 
pausal population. It remains unclear whether Bikram Yoga on its own can combat osteoporosis, but the results of the present study are very promising and warrant further investigation.

\section{Acknowledgements}

This study was supported by Bikram's Yoga College of India in Los Angeles. The contribution of Travis Liggett in the early stage of study in obtaining the Institutional Review Board (IRB) approval is gratefully acknowledged. In addition the contributions of Robert Rude, M.D., Erin Haggerty, M.D., Maureen Hamid, and Joshua Sy are gratefully acknowledged.

\section{References}

[1] National Osteoporosis Foundation (2011). http://nof.org/learn

[2] Black, D.M., Cummings, S.R., Genant, H.K., Nevitt, M.C., Palermo, L. and Browner, W. (1992) Axial and Appendicular Bone Density Predict Fractures in Older Women. Journal of Bone and Mineral Research, 7, 633-638. http://dx.doi.org/10.1002/jbmr.5650070607

[3] Cummings, S.R., Black, D.M., Nevitt, M.C., Browner, W.S., Cauley, J.A., Genant, H.K., Mascioli, S.R., Scott, J.C., Seeley, D.G., Steiger, P., et al. (1990) Appendicular Bone Density and Age Predict Hip Fracture in Women. The Study of Osteoporotic Fractures Research Group. JAMA, 263, 665-668. http://dx.doi.org/10.1001/jama.1990.03440050059033

[4] Vestergaard, P., Rejnmark, L. and Mosekilde, L. (2009) Loss of Life Years after a Hip Fracture. Acta Orthopaedica, 80, 525-530. http://dx.doi.org/10.3109/17453670903316835

[5] Chan, K., Anderson, M. and Lau, E. (2003) Exercise Interventions: Defusing the World's Osteoporosis Time Bomb. Bulletin of the World Health Organization, 81, 827-830.

[6] Ahola, R., Korpelainen, R., Vainionpaa, A., Leppaluoto, J. and Jamsa, T. (2009) Time-Course of Exercise and Its Association with 12-Month Bone Changes. BMC Musculoskeletal Disorders, 10, 138. http://dx.doi.org/10.1186/1471-2474-10-138

[7] Kemmler, W., von Stengel, S., Engelke, K., Haberle, L. and Kalender, W.A. (2010) Exercise Effects on Bone Mineral Density, Falls, Coronary Risk Factors, and Health Care Costs in Older Women: The Randomized Controlled Senior Fitness and Prevention (SEFIP) Study. Archives of Internal Medicine, 170, 179-185.

http://dx.doi.org/10.1001/archinternmed.2009.499

[8] Michaelsson, K., Byberg, L., Ahlbom, A., Melhus, H. and Farahmand, B.Y. (2011) Risk of Severe Knee and Hip Osteoarthritis in Relation to Level of Physical Exercise: A Prospective Cohort Study of Long-Distance Skiers in Sweden. PLoS One, 6, e18339. http://dx.doi.org/10.1371/journal.pone.0018339

[9] Campion, F., Nevill, A.M., Karlsson, M.K., Lounana, J., Shabani, M., Fardellone, P. and Medelli, J. (2010) Bone Status in Professional Cyclists. International Journal of Sports Medicine, 31, 511-515. http://dx.doi.org/10.1055/s-0029-1243616

[10] Creighton, D.L., Morgan, A.L., Boardley, D. and Brolinson, P.G. (2001) Weight-Bearing Exercise and Markers of Bone Turnover in Female Athletes. Journal of Applied Physiology, 90, 565-570.

[11] Dook, J.E., James, C., Henderson, N.K. and Price, R.I. (1997) Exercise and Bone Mineral Density in Mature Female Athletes. Medicine and Science in Sports and Exercise, 29, 291-296. http://dx.doi.org/10.1097/00005768-199703000-00002

[12] Mudd, L.M., Fornetti, W. and Pivarnik, J.M. (2007) Bone Mineral Density in Collegiate Female Athletes: Comparisons among Sports. Journal of Athletic Training, 42, 403-408.

[13] Nichols, J.F., Palmer, J.E. and Levy, S.S. (2003) Low Bone Mineral Density in Highly Trained Male Master Cyclists. Osteoporosis International, 14, 644-649. http://dx.doi.org/10.1007/s00198-003-1418-z

[14] Mukherjee, A., Mukherjee, P. and Rude, R. (2010) Bikram Yoga as a Countermeasure of Bone Loss in Women. Chinese Medicine, 1, 1-4. http://dx.doi.org/10.4236/cm.2010.11001

[15] Tucker, K.L. (2014) Colas, but Not Other Carbonated Beverages, Are Associated with Low Bone Mineral Density in Older Women: The Framingham Osteoporosis Study. American Journal of Clinical Nutrition, 84, 936-942.

[16] Tucker, K.L. (2003) Dietary Intake and Bone Status with Aging. Current Pharmaceutical Design, 9, 2687-2704. http://dx.doi.org/10.2174/1381612033453613

[17] Chilibeck, P.D., Vatanparast, H., Pierson, R., Case, A., Olatunbosun, O., Whiting, S.J., Beck, T.J., Pahwa, P. and Biem, H.J. (2013) Effect of Exercise Training Combined with Isoflavone Supplementation on Bone and Lipids in Postmeno- 
pausal Women: A Randomized Clinical Trial. Journal of Bone and Mineral Research, 28, 780-793. http://dx.doi.org/10.1002/jbmr.1815

[18] Bonaiuti, D., Shea, B., Iovine, R., et al. (2002) Exercise for Preventing and Treating Osteoporosis in Postmenopausal Women (Cochrane Review). In: The Cochrane Library, Issue 4, Update Software, Oxford,

[19] Fishman, L. (2009) Yoga for Osteoporosis. Topics in Geriatric Rehabilitation, 25, 244-250. http://dx.doi.org/10.1097/TGR.0b013e3181b02dd6

[20] Woo, J., Hong, A., Lau, E. and Lynn, H. (2007) A Randomised Controlled Trial of Tai Chi and Resistance Exercise on Bone Health, Muscle Strength and Balance in Community-Living Elderly People. Age and Ageing, 36, 262-268. http://dx.doi.org/10.1093/ageing/afm005

[21] Wu, X.P., Liao, E.Y., Huang, G., Dai, R.C. and Zhang, H. (2003) A Comparison Study of the Reference Curves of Bone Mineral Density at Different Skeletal Sites in Native Chinese, Japanese, and American Caucasian Women. Calcified Tissue International, 73, 122-132. http://dx.doi.org/10.1007/s00223-002-1069-7

[22] Del Rio, L., Pons, F., Huguet, M., Setoain, F.J. and Setoain, J. (1995) Anteroposterior versus Lateral Bone Mineral Density of Spine Assessed by Dual X-Ray Absorptiometry. European Journal of Nuclear Medicine, 22, 407-412. http://dx.doi.org/10.1007/BF00839054

[23] Office of the Surgeon General (US) (2004) Bone Health and Osteoporosis: A Report of the Surgeon General. Office of the Surgeon General (US), Rockville. http://www.ncbi.nlm.nih.gov/books/NBK45503/

[24] Oken, B.S., et al. (2006) Randomized, Conrolled, Six-Motn Trail of Yoga in Healthy Seniors: Effects on the Cognition and Quality of Life. Alternative Therapies in Health and Medicine, 12, 40-47.

[25] Woodyard, C. (2011) Exploring the Therapeutic Effects of Yoga and Its Ability to Increase Quality of Life. International Journal of Yoga, 4, 49-54. http://dx.doi.org/10.4103/0973-6131.85485

[26] Vogt, M.T., Cauley, J.A., Kuller, L.H. and Nevitt, M.C. (1997) Bone Mineral Density and Blood Flow to the Lower Extremities: The Study of Osteoporotic Fractures. Journal of Bone and Mineral Research, 12, 283-289. http://dx.doi.org/10.1359/jbmr.1997.12.2.283 